\title{
The Cosmic Production of Helium
}

\author{
Raul Jimenez, ${ }^{1 *}$ Chris Flynn, ${ }^{2,3}$ James MacDonald ${ }^{4}$ and Brad K. Gibson ${ }^{3}$ \\ ${ }^{1}$ Department of Physics and Astronomy, University of Pennsylvania, PA 19104-6396, USA \\ ${ }^{2}$ Tuorla Observatory, Piikkiö, FIN-21500, Finland \\ ${ }^{3}$ Centre for Astrophysics \& Supercomputing, Swinburne University, Victoria, Australia \\ ${ }^{4}$ Department of Physics and Astronomy, University of Delaware, Newark DE 19716, USA \\ *To whom correspondence should be addressed; E-mail: raulj@physics.upenn.edu
}

\begin{abstract}
We estimate the cosmic production rate of helium relative to metals $(\Delta Y / \Delta Z)$ using $K$ dwarf stars in the Hipparcos catalog with accurate spectroscopic metallicities. The best fitting value is $\Delta Y / \Delta Z=2.1 \pm 0.4$ at the $68 \%$ confidence level. Our derived value agrees with determinations from HII regions and with theoretical predictions from stellar yields with standard assumptions for the initial mass function. The amount of helium in stars determines how long they live and therefore how fast they will enrich the insterstellar medium with fresh material.
\end{abstract}

The amount of helium divided by the amount of heavier elements produced in stars $(\Delta Y / \Delta Z)$, is of great interest to astrophysics and cosmology. The ratio governs the stellar clock and thus how long stars will live. Therefore, age determinations of both resolved and integrated stellar populations rely on knowing how helium concentration changes as a function of metallicity. Because ages of galaxies can help to determine the nature of dark energy (1), an accurate determination of $\Delta Y / \Delta Z$ is needed. It also has an impact on the determination of primordial helium abundance by use of extra-galactic HII regions (2). In addition to the above cosmological interest, it is also a test of theoretical predictions of stellar yields, because given an initial mass function for stars, $\Delta Y / \Delta Z$ is a predicted quantity of stellar evolution.

Helium lines can be observed only in stars with an effective temperature greater than $20,000 \mathrm{~K}$; it is not possible to measure helium abundances directly in cooler stars which live more than $10^{8}$ yr. There are two methods to determine observationally the value of $\Delta Y / \Delta Z$ (where $Y$ and $Z$ are the fractional abundances of helium and all metals hevier than helium, respectively). One is through the use of HII extra-galactic regions to measure helium's primordial abundance, which then is compared to that of the Sun to deduce $\Delta Y / \Delta Z$. This is done by either extrapolation of correlations of $Y-(O / H)$ with $Y-(N / H)$ to $O / H=N / H=0$ (e.g. (3] 4] 5) and references 
therein) or by measuring $Y$ in ultra-low metallicity blue compact dwarf galaxies and assuming that no chemical evolution has taken place (e.g. (6)). Recent values for $\Delta Y / \Delta Z$ range from $2.5(7)$ to $3.5(8)$, which agrees with stellar yield computations assuming a standard Salpeter, Scalo or Kroupa initial mass function (IMF) (9, 10).

The other (indirect) method exploits the fact that the temperature of main sequence dwarf stars is insensitive to stellar age. The strongest dependences are on $Y, Z$ (which can be determined from spectroscopy) and on the value of the mixing length parameter. The mixing length parameter can be calibrated in stellar models using the Sun and the very thin and narrow red giant branches of globular clusters indicate that other stars have the same mixing length as the Sun. Further, the fact that all globular clusters require the same value for the mixing length parameter supports the supposition that the mixing length parameter does not depend on $Z$ (e.g. (11)). Although rotation may affect the temperature of a main sequence star, its effect is small (12). Therefore, once the metallicity has been measured, the color and luminosity of the main sequence depends only on the value of $Y$. Because the isochrone for the Sun is a fixed point (the $Y$ value is adjusted to match the present age and radius of the Sun), the effect of varying $\Delta Y / \Delta Z$ anti-correlates with the broadening of the main sequence stellar values. Here we apply a related method to that employed by (13) and newly available spectroscopic metallicities of stars in the Hipparcos sample to put tighter constraints on the value of $\Delta Y / \Delta Z$.

This method of determining $\Delta Y / \Delta Z$ has been applied to stars in the solar neighborhood using ground-based parallaxes. (14) found a value $\Delta Y / \Delta Z=5 \pm 3$, (12) found $\Delta Y / \Delta Z>2$. Recently, (13) used the Hipparcos parallaxes, infrared flux temperatures and accurate, spectroscopically determined metallicities for sub-solar metallicity stars. They derived a value $\Delta Y / \Delta Z=3 \pm 2$.

The sample consists of K dwarfs for which we have accurate Hipparcos parallaxes, accurate spectroscopically determined metallicities, and broad band in filters $V$ and $B$. Our primary sources of metallicities are $\mathrm{G}$ and $\mathrm{K}$ dwarfs in (15, 16 17 18 19). No binaries or suspected binaries were used. The complete list of sample stars is shown in Table 1.

We only use stars in the absolute magnitude range $5.5<M_{V}<7.5$. The limit at $M_{V}=$ 5.5 ensures the stars have sufficiently low mass that no significant stellar evolutionary effects have taken place since they arrived on the main sequence; stars more luminous than this limit have begun to evolve off the main sequence, meaning that their luminosities are no longer primarily dependent on $Z$ and $Y$. Stars fainter than $M_{V}=7.5$ are excluded because metallicity determinations for these cooler, lower mass stars are still difficult to perform because of their complex spectra. The $B-V$ colors mainly come from (20), our own photometry (21) and the Hipparcos catalog.

The absolute magnitude of a star relative to the luminosity of the solar metallicity isochrone at the same $B-V$ color is defined as $\Delta M_{V}$ (figure 1). This quantity is tightly correlated with metallicity, as first shown in (21), as seen in figure 2.

For a fixed $Z$, an increase in $Y$ translates into an increase of luminosity and effective temperature due to the increase in molecular weight. On the other hand, an increase in $Z$ produces a dimming and cooling of the star due to the presence of more metals. Although this behav- 
ior can be understood by using quasi-homology relations which are derived for fully radiative cores (e.g. (12)), it is not useful because dwarfs along the main sequence have rather deep convective envelopes. We have used the stellar evolution code JMSTAR (22, 23, 24) to compute isochrones for $5.5<M_{V}<7.5$ and $0.4<B-V<1.4$, or equivalently effective temperatures, $6000<T_{\text {eff }}<4500$. We use of opacities from (25) for high $Z$ stars, because otherwise these stars would be too blue (26). We used the code to compute stellar tracks on the main sequence for stars with masses $0.6<M / M_{\odot}<1.2$. We have computed isochrones from the above tracks and then converted them from the theoretical $\left(L-T_{\text {eff }}\right)$ to the observational plane $\left(M_{V}-(B-V)\right)$ using the most recent Kurucz atmospheres models ${ }^{1}$.

We use the fiducial solar isochrone (Fig. 1) as our reference point to measure $\Delta M_{V}$ for the model stars at a fixed $B-V$ color. The locus of the stars in the $\Delta M_{V}$ versus $\log Z$ plane shows a correlation between luminosity and metallicity that is consistent with the theoretical stellar evolutionary predictions for isochrones with $\Delta Y / \Delta Z=1,2$ and 4 . While the luminosity of the stars is primarily sensitive to metallicity, we also confirm our expectation that the effect of $Y$ is only seen when the metallicity is sufficiently high $\left(Z / Z_{\odot}>0.3\right)$. Note that we have chosen the primordial abundance to be $Y_{p}=0.236(4)$, so that at $Z / Z_{\odot}=0.3$ the corresponding values of $Y$ are $0.24,0.25$ and 0.26 for $\Delta Y / \Delta Z=1,2$ and 4, respectively: i.e. these are sufficiently different in $Y$ to start to have an effect on the luminosity of the star. We have chosen to fix $Y_{p}$ and then compute the corresponding $Y$ by using $Y=Y_{p}+\frac{\Delta Y}{\Delta Z} Z$, instead of what (13) do, which is to compute $Y$ using the Sun as the anchor point. The results of (13) lead to primordial helium abundance of 0.26 and 0.20 for $\Delta Y / \Delta Z=1$ and 4 , respectively which are inconsistent with values inferred from HII regions $(3|6| 4 \mid 5)$. Even if the primordial value has been enhanced due to the presence of a primordial zero metallicity population with very massive stars (up to 1000 $\mathrm{M}_{\odot}$ ), the effect will be very small (28), only about 0.01 , which does not affect our conclusions.

The isochrones with $\Delta Y / \Delta Z=4$ are not favored by our analysis. To obtain the statistical significance of the best fitting model, we have performed a maximum likelihood fit of the data to the models. Because the errors are Gaussian distributed and uncorrelated, this translates into simple $\chi^{2}$ statistics. We found the best $\chi^{2}$ by minimizing the distance of the observational points to the models (Fig. 3). The reduced $\chi^{2}$ for $\Delta Y / \Delta Z=2$ is 1.2 , which indicates that these models are reasonable fit. The probability of this value of $\chi^{2}$ being the correct model, using the incomplete gamma function of the number of degrees of freedom and the value of $\chi^{2}$, is about $20 \%$. We computed 4 models for $\Delta Y / \Delta Z=1,2,3$ and 4 and fitted a Gaussian to the likelihood, which is a parabola in $\chi^{2}$, to these points (Fig. 3). Both values 1 and 4 are excluded at greater than the $2 \sigma$ level. The best fit corresponds to $\Delta Y / \Delta Z=2.1 \pm 0.4$. Which represents a much narrower range than previous studies. The low metallicity part $\left(\log _{10} Z<-2.4\right)$ of the sample can be used to determine the value of primordial helium $Y_{p}$, because this part is not sensitive to $\Delta Y / \Delta Z$ (Fig. 2). A maximum likelihood fit produces $Y_{p}=0.24 \pm 0.02$, which is consistent with our chosen value and those derived from HII regions but with a larger

\footnotetext{
${ }^{1}$ Available at http:/ / cfaku5.harvard.edu/grids.html Recently, (27) have studied the reliability of these models, concluding they do an excellent job at predicting the correct color $-T_{\text {eff }}$ relation when compared with other methods (e.g the infrared flux method).
} 
error (3] 6. 4[5) .

The dominant systematic uncertainty plaguing our analysis can be traced to the inherent assumption of solar-scaled abundances in both the data and theoretical stellar atmospheres. Only a small fraction of the super-solar metallicity K-dwarfs in the solar neighborhood are enhanced in $\alpha$-elements with respect to iron (29). The $\alpha$-enhanced isochrones of (30) demonstrate that for every 0.2 dex increase in $[\alpha / \mathrm{Fe}]$ above the scaled-solar ratio, $\Delta \mathrm{M}_{\mathrm{V}}$ increases by $\sim 0.07 \mathrm{mag}$. Our sample does not have such a high enhancement; multi-species abundances (16.17) show a maximum value of 0.05 with an average value of zero, consistent with no enhancement. As such, we assign a conservative systematic error budget on $\Delta \mathbf{M}_{\mathrm{V}}$ of ${ }_{-0.02}^{+0.0}$. On the contrary, low metallicity stars have $[\alpha / \mathrm{Fe}] \sim 0.4$. For metal-poor stars the effect of alpha enhancement can be accurately approximated by scaling to a higher metallicity the solar-scaled isochrones (13). The effect this has on an isochrone is to make it cooler but also brighter (since we are measuring $\Delta M_{v}$ at a fixed color). These two compensate each other and the net effect is that the points slide along the solar-scaled isochrones of figure 2 .

We derive $\Delta Y / \Delta Z=2.1 \pm 0.4$ at the $68 \%$ confidence level. Our $\Delta Y / \Delta Z$ agrees with modern determinations from HII regions $\left(Y_{p}=0.236\right)(645)$ and the helium abundance needed to fit the Sun with its current age and radius $\left(Y_{\odot}=0.275, Z_{\odot}=0.017\right)$, which gives $\Delta Y / \Delta Z=$ 2.3. It also agrees with theoretical predictions from stellar yields with standard assumption for the initial mass function (9]10), which translates into a upper mass cut-off of $100 \mathrm{M}_{\odot}$ for a Salpeter IMF. But, is not consistent with $\Delta Y / \Delta Z$ derived for the Hyades (31), which is $<1$. If the Hyades value is confirmed, it will indicate a different evolutionary path in the chemical enrichment in the Milky Way.

\section{References}

1. R. Jimenez, A. Loeb, ApJ 573, 37 (2002).

2. M. Peimbert, S. Torres-Peimbert, ApJ 203, 581 (1976).

3. Y. I. Izotov, T. X. Thuan, V. A. Lipovetsky, ApJS 108, 1 (1997).

4. A. Peimbert, M. Peimbert, V. Luridiana, ApJ 565, 668 (2002).

5. R. Gruenwald, G. Steigman, S. M. Viegas, ApJ 567, 931 (2002).

6. Y. I. Izotov, F. H. Chaffee, R. F. Green, ApJ 562, 727 (2001).

7. T. X. Thuan, Y. I. Izotov, Space Science Reviews 100, 263 (2002).

8. M. Peimbert, A. Peimbert, M. . Ruiz, ApJ 541, 688 (2000).

9. A. Maeder, $A \& A$ 264, 105 (1992). 
10. T. Tsujimoto, et al., ApJ 483, 228 (1997).

11. R. Jimenez, P. Thejll, U. G. Jorgensen, J. Macdonald, B. Pagel, MNRAS 282, 926 (1996).

12. J. Fernandes, Y. Lebreton, A. Baglin, $A \& A$ 311, 127 (1996).

13. B. E. J. Pagel, L. Portinari, MNRAS 298, 747 (1998).

14. M.-N. Perrin, G. C. de Strobel, R. Cayrel, P. M. Hejlesen, A\&A 54, 779 (1977).

15. C. Flynn, O. Morell, MNRAS 286, 617 (1997).

16. P. Thorén, S. Feltzing, $A \& A$ 363, 692 (2000).

17. S. Feltzing, G. Gonzalez, $A \& A$ 367, 253 (2001).

18. B. Chaboyer, P. Demarque, P. J. Kernan, L. M. Krauss, ApJ 494, 96 (1998).

19. J. Tomkin, D. L. Lambert, ApJ 523, 234 (1999).

20. M. S. Bessel, $A \& A S$ 83, 357 (1990).

21. E. Kotoneva, C. Flynn, R. Jimenez, MNRAS 335, 1147 (2002).

22. R. Jimenez, J. Macdonald, MNRAS 283, 721 (1996).

23. D. J. Mullan, J. MacDonald, ApJ 559, 353 (2001).

24. T. Lawlor, J. MacDonald, ApJ 583, 000 (2003).

25. D. Alexander, J. Ferguson, ApJ p. 879 (1994).

26. V. Castellani, S. Degl'Innocenti, P. G. Prada Moroni, MNRAS 320, 66 (2001).

27. M. S. Bessell, F. Castelli, B. Plez, $A \& A$ 333, 231 (1998).

28. P. Marigo, C. Chiosi, R. P. Kudritzki, astro-ph/0212057 (2002).

29. B. J. Taylor, apjs 22, 177 (1970).

30. B. Salasnich, L. Girardi, A. Weiss, C. Chiosi, A\&A 361, 1023 (2000).

31. Y. Lebreton, J. Fernandes, T. Lejeune, $A \& A$ 374, 540 (2001).

32. RJ is supported by NSF grant AST-0206031. BKG \& CF acknowledge the support of the Australian Research Council through its Large Research Grant (A010517), Discovery Project (DP0343508), and Linkage International Award (LX0346832) schemes. This research was supported by the Academy of Finland through its funding of the ANTARES program for space research. We have made extensive use of the Simbad stellar data base, at the Centre de Données astronomiques de Strasbourg, for which we are very grateful. 


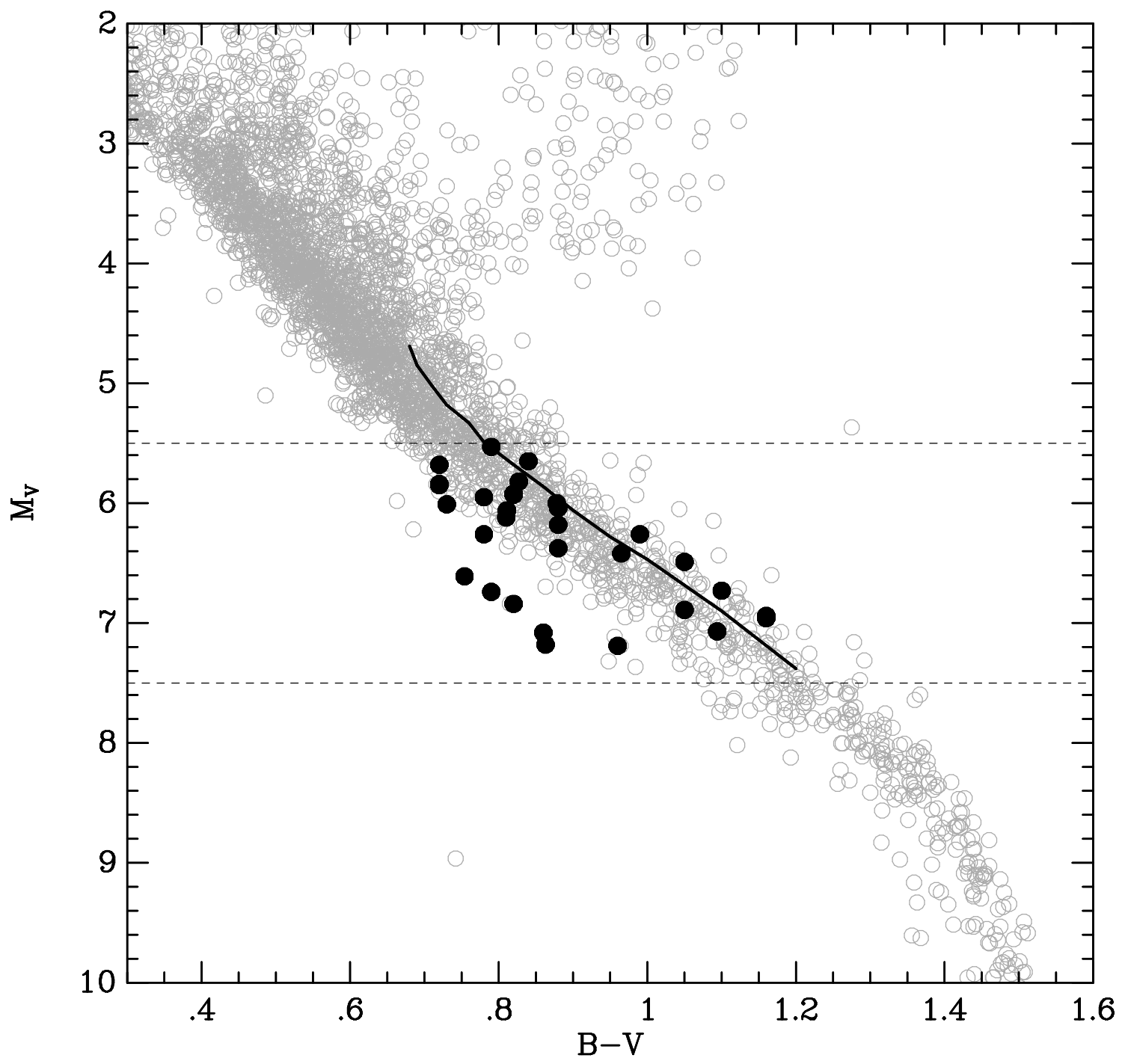

Figure 1: Our sample of $\mathrm{K}$ dwarf stars (solid symbols) plotted over the Hipparcos colormagnitude diagram (open symbols). The reference solar isochrone is shown by the solid line. The difference between a star's luminosity $M_{V}$ and the luminosity of the isochrone at the same color is the quantity $\Delta M_{V}$, which correlates tightly with the stellar metallicity in figure 2 . Note that in plotting Hipparcos stars (open symbols), only non-multiple stars with parallax errors smaller than $5 \%$ and color errors less than 0.02 magnitudes have been used. There are a small number of stars a few tenths of a magnitude above the main sequence which are likely to be unrecognized binaries. 


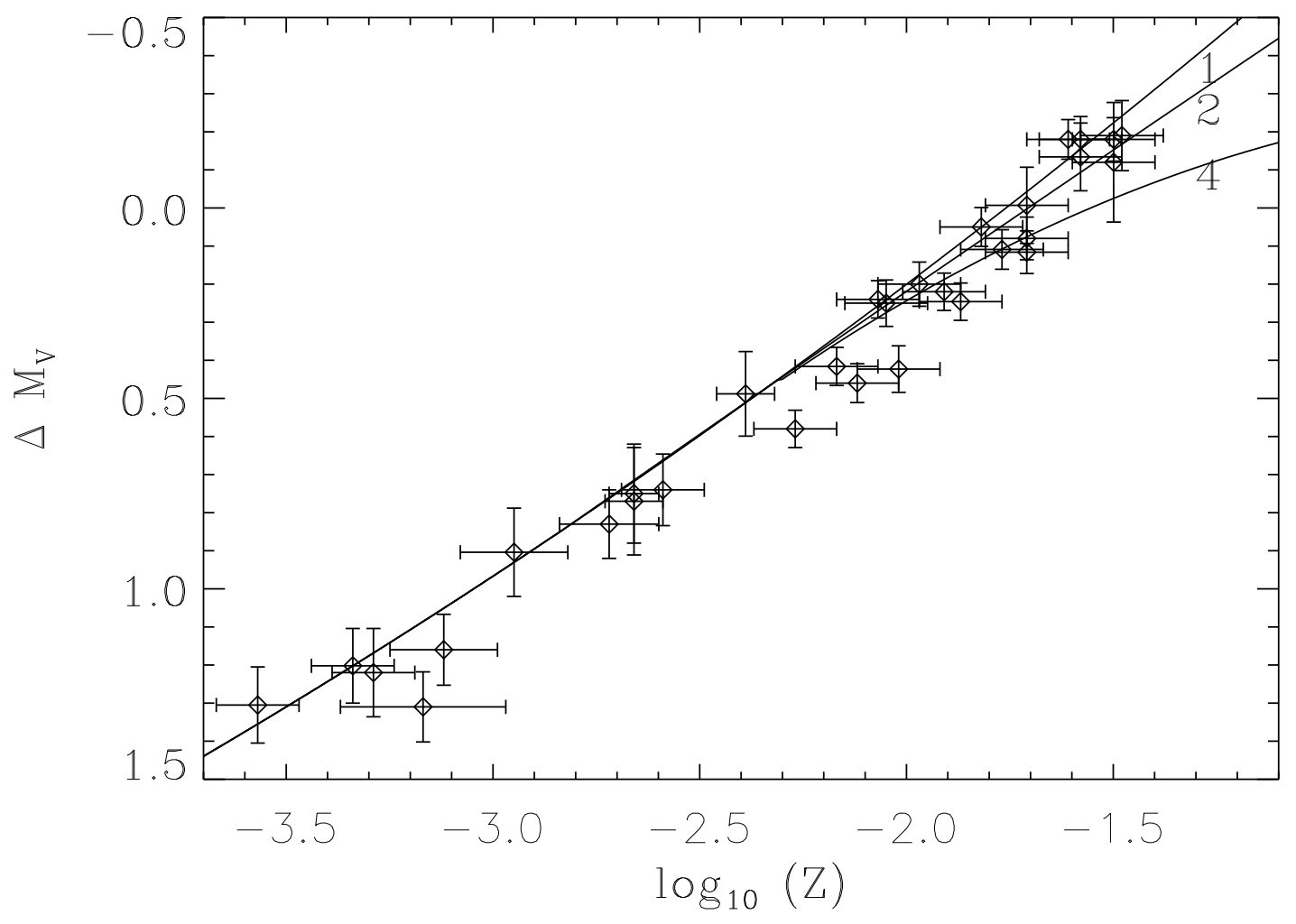

Figure 2: The solid lines correspond to $\Delta M_{V}$, measured from the fiducial solar isochrone, from theoretical models for $\mathrm{K}$ dwarfs for different values of $\Delta Y / \Delta Z$ (1,2 and 4 from top to bottom) as a function of metallicity. The observational points are for $\mathrm{K}$ dwarfs with Hipparcos parallaxes and with spectroscopically determined metallicities. The errors in $\Delta M_{V}$ are driven by the color and parallax errors in about equal proportion. The spectroscopically determined metallicities have errors of typically 0.1 dex. 


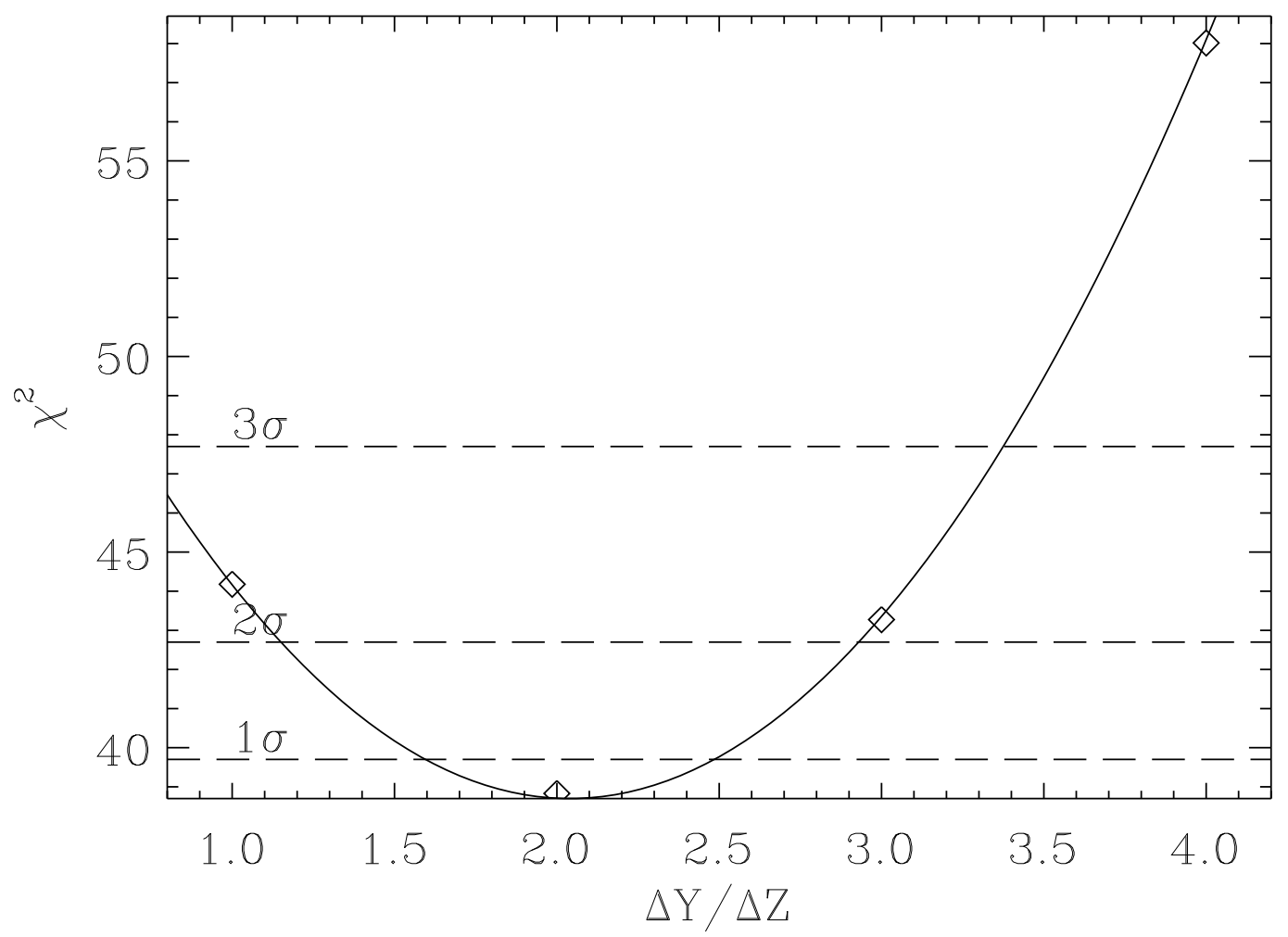

Figure 3: $\chi^{2}$ for four models (diamonds) and the data of figure 2. The solid line is a parabola fitted to the models. The dashed lines represent several confidence levels. Note that values as low as 1 and as high as 3 are excluded at more than $95 \%$ confidence. 
Table 1: The sample of Hipparcos K dwarfs with spectroscopic metallicities. The final column "S" indicates the sources of the metallicities. The codes are: 1: Tomkin and Lambert (1999). 2: Thoren and Feltzing (2000), Feltzing and Gonzalez (2001). 3: Chaboyer et al (1998). 4: Flynn and Morell (1997). 5: Cayrel catalog.

\begin{tabular}{lrrrrrr}
\hline Name & $V$ & $B-V$ & $M_{V}$ & {$[\mathrm{Fe} / \mathrm{H}]$} & $\Delta M_{V}$ & $\mathrm{~S}$ \\
\hline HD 4628 & 5.74 & 0.88 & 6.38 & -0.40 & 0.42 & 4 \\
HD 10700 & 3.53 & 0.72 & 5.68 & -0.50 & 0.58 & 4 \\
HD 13445 & 6.12 & 0.82 & 5.93 & -0.28 & 0.25 & 4 \\
HD 21197 & 7.86 & 1.16 & 6.96 & 0.27 & -0.23 & 2 \\
HD 22049 & 3.72 & 0.88 & 6.18 & -0.14 & 0.22 & 1 \\
HD 25329 & 8.50 & 0.86 & 7.18 & -1.80 & 1.30 & 5 \\
HD 26965 & 4.41 & 0.82 & 5.92 & -0.30 & 0.24 & 4 \\
HD 30501 & 7.58 & 0.88 & 6.04 & 0.06 & 0.08 & 2 \\
HD 31392 & 7.60 & 0.79 & 5.53 & 0.06 & -0.01 & 2 \\
HD 32147 & 6.22 & 1.05 & 6.49 & 0.29 & -0.19 & 4 \\
HD 61606 & 7.18 & 0.96 & 6.42 & 0.06 & 0.08 & 2 \\
HD 64606 & 7.43 & 0.73 & 6.01 & -0.95 & 0.83 & 1 \\
HD 65583 & 6.97 & 0.72 & 5.84 & -0.82 & 0.74 & 1 \\
HD 72673 & 6.38 & 0.78 & 5.95 & -0.35 & 0.46 & 4 \\
HD 87007 & 8.82 & 0.84 & 5.65 & 0.27 & -0.12 & 2 \\
HD 100623 & 5.96 & 0.81 & 6.06 & -0.25 & 0.42 & 4 \\
HD 103095 & 6.45 & 0.75 & 6.61 & -1.40 & 1.31 & 3,4 \\
HD 103932 & 6.99 & 1.16 & 6.94 & 0.16 & -0.25 & 2 \\
HD 108564 & 9.45 & 0.96 & 7.19 & -1.18 & 0.87 & 1 \\
HD 134439 & 9.10 & 0.79 & 6.74 & -1.57 & 1.20 & 4 \\
HD 134440 & 9.45 & 0.86 & 7.08 & -1.52 & 1.22 & 4 \\
HD 136834 & 8.28 & 0.99 & 6.26 & 0.19 & -0.17 & 2 \\
HD 145417 & 7.53 & 0.82 & 6.84 & -1.35 & 1.16 & 5 \\
HD 149661 & 5.76 & 0.83 & 5.82 & 0.00 & 0.11 & 4 \\
HD 192031 & 8.67 & 0.72 & 5.85 & -0.89 & 0.75 & 1 \\
HD 192310 & 5.73 & 0.88 & 6.00 & -0.05 & 0.05 & 4 \\
HD 209100 & 4.69 & 1.05 & 6.89 & -0.10 & 0.21 & 4 \\
HD 213042 & 7.67 & 1.10 & 6.73 & 0.19 & -0.17 & 2 \\
HD 216803 & 6.48 & 1.09 & 7.07 & -0.20 & 0.20 & 4 \\
BD +41 3306 & 8.86 & 0.81 & 6.12 & -0.62 & 0.49 & 1 \\
BD +24 4460 & 9.51 & 0.78 & 6.26 & -0.89 & 0.77 & 1 \\
\hline
\end{tabular}

